\title{
Pelvic Floor Dysfunction and Management: Comprehensive Review
}

\author{
S. Manimegalai \\ Department Obstetrics and Gynecological Nursing, College of Nursing Sciences, Dayananda Sagar University, Kumaraswamy Layout, Bengaluru, Karnataka, India
}

\section{Abstract}

The pelvic floor is a complex, multilayered group of muscles and surrounding tissue that are suspended like a hammock between the pubic bone in front and the base of the spinal column in the back. Pelvic floor dysfunction is a disorder predominantly affecting females. It is common and affects the quality of lives at least one-third of adult women and is a growing component of women's health care needs. At present, pelvic floor dysfunction is becoming a major problem during the whole process of childbirth. A study was conducted and shows that the overall prevalence of post-natal urinary incontinence is $6-32 \%$ and fecal incontinence is $13-25 \%$. Hence, pelvic floor dysfunction can have a devastating effect on women's life. Pelvic floor dysfunction causes many changes, physically and mentally. Hence, prevention is better than cure. Maintaining healthy pelvic floor muscles by doing pelvic floor muscle exercise will prevent the risk of disease. Hence, the nurse-midwife plays a key role within the multidisciplinary team to help improve the quality of life for women by offering conservative management and prophylactic measures during the post-natal period to prevent complications. Although puerperium is a natural physiological process, it should be recognized as a challenge to prevent complications during puerperium. Health care workers help the woman to adapt with home care management to prevent complications so, that the woman will be better equipped to prevent the complications. This article proposes definitions, causes, types, symptoms, effects, preventive measures, and management of pelvic floor dysfunction.

Keywords: Pelvic floor, Management, Dysfunction

\section{INTRODUCTION}

The post-natal period refers to the 6 weeks period after childbirth. In this period, the pelvic organs revert back approximately to the pre-pregnant state both anatomically and physiologically are known as involution of pelvic structures. Women can experience problems during the post-natal period such as tiredness, perineal pain, breast problems, backache, hemorrhoids, constipation, depression, anemia, headache, and pelvic floor dysfunction. At present, pelvic floor dysfunction is becoming a major problem during post-natal period. Pelvic floor dysfunction is a condition where pelvic floor muscles lose its control and relax completely following childbirth which alters the normal bladder and bowel functions during the post-natal period. The pelvic floor or pelvic diaphragm is

Access this article online

Website: http://innovationalpublishers.com/Journal/ijnmi

ISSN No: 2656-4656

DOI: $10.31690 /$ ijnmi.2020.v05i03.003 composed of muscle fibers of the levator ani, the coccygeus, and associated connective tissue which span the area underneath the pelvis. The functions of the pelvic floor are providing support of abdominopelvic viscera (bladder, intestines, uterus, etc.) through their tonic contraction, maintaining resistance to increases in intra-pelvic/abdominal pressure during activities such as coughing or lifting heavy objects and the muscle fibers have a sphincter action on the rectum and urethra. They relax to allow urination and defecation. ${ }^{[1]}$

\section{Definition Of Pelvic Floor Dysfunction}

Pelvic floor dysfunction is the inability to control the muscles of the pelvic floor. ${ }^{[2]}$

\section{Causes Of Pelvic Floor Dysfunction During Puerperium}

Multiparity, it is one of the main causes of pelvic floor disorders. A woman's risk tends to increase the more times she 
has given birth. ${ }^{[3]}$ Intra-natal interventions such as continuous electronic fetal monitoring to keep track of the baby's heart rate (being connected to this monitor throughout labor vs. monitoring at regular intervals), using of epidural analgesia for relieving labor pain (vs. many other drugs and drug-free measures for pain relief), delivering the baby by vacuum extraction or forceps delivery, providing the position such as lying on the back (supine position) or on the back with legs in stirrups (lithotomy position) for pushing, and giving birth this works against gravity, yet is used with most births in the U.S. providing episiotomy: Cutting the back of vaginal opening to enlarge it for birth, Caregiver-directed pushing or purple pushing: In which women are directed to bear down as long and hard as they can during contractions once the cervix is fully dilated. This common practice can be far more forceful than when a woman's own natural pushing reflexes move the baby out, fundal pressure: A member of the medical team presses on the woman's abdomen to help move the baby out. ${ }^{[3]}$ During pregnancy, the extra weight and pressure of the baby, the "bag of waters," and the placenta causes many women to experience urinary incontinence by the end of pregnancy. ${ }^{[3]}$

\section{Types Of Pelvic Floor Dysfunction}

The three main types of pelvic floor disorders are: Urinary incontinence or lack of bladder control: Overflow incontinence: It is a form of urinary incontinence characterized by the involuntary release of urine from an overfull urinary bladder, often in the absence of any urge to urinate. ${ }^{[4]}$ Stress urinary incontinence: This is a bladder control problem that occurs when urine leaks during physical activities. When stress is placed on the bladder during laughter or physical activity, pressure in the abdomen rises, often resulting in leakage in the form of spurts of urine. Urge incontinence or overactive bladder: This occurs when a woman has a sudden and strong urge to urinate and leaks on herself before she can get to the toilet. Fecal incontinence or lack of bowel control: ${ }^{[4]}$ It is also known as anal incontinence is the lack of control over defecation, leading to involuntary loss of bowel contents including flatus, liquid stool elements and mucus, or solid feces. Pelvic organ prolapse: It is a condition in which the uterus, bladder and bowel "drop" into the vagina and cause a bulge through the vaginal canal. ${ }^{[4]}$ Types of pelvic organ prolapse include cystocele: The bladder protrudes through the vagina, enterocele: The small intestine protrudes through the upper vaginal supports (especially in women who have had a hysterectomy), rectal prolapse: The lining and/or muscles of the rectum protrude out of the anus, typically during attempts at bowel movements, rectocele: The rectum protrudes through the back wall of the vagina, sigmoidocele: The sigmoid colon (the part of the large intestine that is closest to the rectum and anus) slips into the rectovaginal space, and vaginal vault prolapse or uterine prolapse: The entire vagina or uterus sag through the vaginal opening female sexual dysfunction, sexual problems such as pain during intercourse and vaginal dryness are often related to pelvic floor disorders that prevent the women from experiencing satisfaction from the sexual activity. ${ }^{[5]}$

\section{Symptoms Associated With Pelvic Floor Dysfunction During Puerperium}

The following are some of the more common problems associated with PFD pelvic pain: Ongoing pain in the pelvic region, genitals, or rectum, chronic in nature, which often worsens with bladder filling; bladder dysfunction: Frequent need to urinate, urinary urgency, and painful urination, and bowel dysfunction: The feeling that needs to have several bowel movements during a short period of time. Dyspareunia: Recurrent genital pain that occurs just before, during or after intercourse. ${ }^{[6]}$

\section{Diagnostic Evaluation}

1. History collection: It must focus on to rule out the cause and symptoms of pelvic floor dysfunction, so while collecting history, questions related to causes and symptoms must be asked from the female include: Do you have a history of urinary tract infections? Have you given birth to a child? Do you have interstitial cystitis (long-term inflammation of the bladder wall) or irritable bowel syndrome (a disorder of the lower intestinal tract)? Do you have pain with intercourse? Are you a multipara mother? Have you undergone for episiotomy or forceps or vacuum extraction during delivery ${ }^{[7]}$

2. Physical examination: Physical examination to evaluate the ability to control pelvic floor muscles. Using hands, check for muscle spasms, muscle knots, or muscle weakness. Clinical examination is the basis of the diagnosis of urogynecological disorders. It is important that this examination is performed by a well-trained person with the appropriate skills. The patient should actively participate in the examination and be able to carry out pelvic floor muscle (PFM) contractions in a coordinated way when required. This will form the basis of subsequent pelvic floor exercises and is how the woman will learn the different types of muscle contraction which are integral to this. ${ }^{[8]}$ Observation method: This qualitative measure can detect an in drawing of the anus, lifting of the posterior vaginal wall, and narrowing of the vaginal introitus (females). ${ }^{[9]}$

3. Perinea meter: Pelvic muscle control is tested by placing electrodes on the perineum (the area between the vagina and rectum in the woman) or sacrum (the triangular bone at the base of the spine). A small device called a perineometer may also be placed into the rectum or vagina to test the muscle control. Advantages: Relatively inexpensive. Able to measure strength and endurance. Quantitative: It can be used when the patient is standing. - Disadvantages: Unable to distinguish right from left. Pressure changes may be caused by an increase in intraabdominal pressure due to the co-contraction 
of the abdominal muscles. No a gold standard at device; different results with different probe sizes and materials. ${ }^{[10]}$

4. Defecating proctogram: Defecography (also known as proctography, defecating/defecation proctography, evacuating/evacuation proctography, or dynamic rectal examination) is a type of medical radiological imaging in which the mechanics of a patient's defecation are visualized in real-time using a fluoroscope. The anatomy and function of the anorectum and pelvic floor can be dynamically studied at various stages during defecation. For this test, the female will be given an enema of a thick liquid that can be detected with an X-ray. A special video X-ray is used to record the movement of the muscles as the mother's attempt to push the liquid of the rectum, or in females, preprocedural preparation involves smearing a small amount of barium contrast agent in the vagina, which will help to identify if anterior rectocele, enterocyte, or sigmoidocele is present. The technique itself involves the insertion of a caulk gun device into the rectum with a subsequent manual infusion of barium paste until there is adequate distension. The patient is then transferred to a portable plastic commode which is situated next to a fluoroscope which records the defecation. Positioning of the X-ray camera is of paramount importance as visualization of the buttocks, rectal vault, and lower pelvis is critical. ${ }^{[8]}$

5. Uroflow test: In the uroflow test, as the mother try to void her bladder. If her flow of urine is weak, it can suggest pelvic floor dysfunction. Uroflowmetry measures the flow of urine. It tracks how fast urine flows, how much flows out, and how long it takes. It is a diagnostic test to assess how well the urinary tract functions. The doctor may suggest uroflowmetry if patients have trouble urinating or have a slow stream. For this test, the patient should arrive at the doctor's office with a fairly full bladder. If possible, do not urinate for a few hours before the test. The patient will be asked to urinate into a funnel connected to the electronic uroflowmeter. This records information about the urine flow on a flow chart. The flow rate is calculated as milliliters $(\mathrm{ml})$ of urine passed per second. Both average and top flow rates are measured. The fastest flow rate, also known as Qmax, is used to understand if a block or obstruction is severe. The average range closer to $15-18 \mathrm{ml} / \mathrm{s}$. A slow or low flow rate may mean there is an obstruction at the bladder neck or in the urethra or a weak bladder. A fast or high flow rate may mean there are weak muscles around the urethra or urinary incontinence problems. ${ }^{[5]}$

6. Laboratory test: Diagnostic testing is performed to determine the best approach to treating pelvic floor dysfunction. Urinalysis: A urine sample is tested for signs of bladder infection, blood in the urine, and other health conditions. Office cystoscopy - a small gentle scope is inserted through the urethra, is used by the doctor to magnify, and examine the inside lining of the bladder and urethra. Post-void measurement - this test measures the amount of urine left in the bladder after urination. The amount of leftover (residual) urine can be measured by draining the bladder with a thin, flexible tube (catheter) or using ultrasound. The catheter method has a slight risk of causing infection or injury to the tube leading from the bladder (urethra). However, the catheter method is safe when done carefully. Moreover, it may be less expensive than ultrasound. ${ }^{[9]}$

7. Pelvic examination - specifically focused on assessing prolapse or other anatomic findings at rest and with straining. The internal genitalia should be examined for estrogen deficiency, abnormal vaginal secretions or urine, pelvic organ prolapse, and abnormal pelvic masses. The anterior, superior, and posterior segments of the vagina should be examined for pelvic organ prolapse. The patient can confirm the examiner has identified the extent of prolapse she experiences. When the patient tells you she normally has a greater amount of prolapse that you presently see, you should have her stand erect and perform any provocative maneuvers which normally are associated with her symptoms. After a period of time, repeat the examination while the patient is standing. In all women, a digital rectal examination is also performed to assess sphincter tone (both resting and active) and to detect fecal impaction or a rectal mass. ${ }^{[4]}$

\section{Conclusion}

Pelvic floor dysfunction causes many changes, physically and mentally. Hence prevention is better than cure. Maintaining healthy pelvic floor muscles by doing pelvic floor muscle exercises will prevent the risk of disease. The family and community have an important role along with the health personnel in the prevention and control of pelvic floor dysfunction during puerperium. The post-natal mothers must have adequate knowledge about pelvic floor dysfunction and its prevention to lead a peaceful postpartum life.

\section{RefEREnCES}

1. Dutta DC. Text Book of Gynecology. $7^{\text {th }}$ ed. New Delhi: Jaypee Publishers; 2016. p. 13, 165, 324, 359.

2. Frota IP, Rocha AB, Neto JA, Vasconcelos CT, de Magalhaes TF, Karbage SA. Pelvic floor muscle function and quality of life in postmenopausal women with and without pelvic floor dysfunction. Acta Obstet Gynecol Scand 2018;97:552-9.

3. Bhargava VL. Text Book of Gynaecology. $2^{\text {nd }}$ ed. New Delhi: ANE Books Private Limited Publishers; 2012. p. 285-95.

4. Malhotra N, Malhotra J, Saxena R, Bora NM. Jeffcott's Principles of Gynaecology. $9^{\text {th }}$ ed. New Delhi: Jaypee Health Science Publishers; 2019. p. 1045-52.

5. Pal A. Practical Manual of Gynecology. $1^{\text {st }}$ ed. New Delhi: Jaypee Brother's Medical Publishers; 2013. p. 170-4.

6. Bradley CS. Prolapse, pain, and pelvic floor muscle dysfunction. Am J Obstet Gynecol 2019;221:177-8. 
7. Bobak IM, Jensen MD, Lowdermilk DL. Maternity Nursing. $4^{\text {th }}$ ed. Missouri: Mosby Publishers; 1995. p. 442.

8. Blomquist JL, Carroll M, Muñoz A, Handa VL. Pelvic floor muscle strength and the incidence of pelvic floor disorders after vaginal and cesarean delivery. Am J Obstet Gynecol 2019;222:62.e1-8.

9. Jacob A. A Comprehensive Text Book of Midwifery and Gynecological
Nursing. $5^{\text {th }}$ ed. New Delhi: Jaypee Publishers; 2019. p. 626-8, 639-40. 10. Beth Shelly PT. The pelvic floor. J Womens Health Phys Therap 2006;30:42.

How to cite this article: Manimegalai S. Pelvic Floor Dysfunction and

Management: Comprehensive Review. Int J Nurs Med Invest. 2020;5(3):38-41. 